\title{
Hybrid energy management with respect to a hydrogen energy system and demand response
}

\begin{abstract}
A Hybrid Energy System (HES) is a mechanism that combines multiple sources of energy connected together to achieve synchronised energy output. However, increased energy consumption, operator energy expenses, and the potential environmental impact of increased emissions from the exhaustion of non-renewable energy resources (fossil fuel) pose major challenges to HES. This research is to conduct energy management strategy based on a demand response (DR) program and a hydrogen storage system by designing a Program Logic Controller (PLC) unit. The hybrid system is evaluated by comparing different scenarios such as a hydrogen energy system and demand response. The purpose of this research is to reducing peak demand, minimise the cost of the system and also to extract surplus power generation out of the rate of the battery. This can be achieved by improving the system performances and by eliminating any degradation at the early stages. Organisations or companies must be sure their systems are working properly and that their investments will pay off.
\end{abstract}

Keyword: Energy management; Hybrid energy; Hydrogen energy; PLC and demand response 